loops, as shown in Fig. 1. A 1, 1, 0 plane also shows an eight 'looped' figure, but in this case the pattern is no longer symmetrical but appears as Fig. 1 would appear if the page was stretched to $\sqrt{2}$ of its present width. This, as well as the fact that a 1, 1, 1 plane shows a six 'looped' figure, is to be expected from the atomic spacing in these planes.

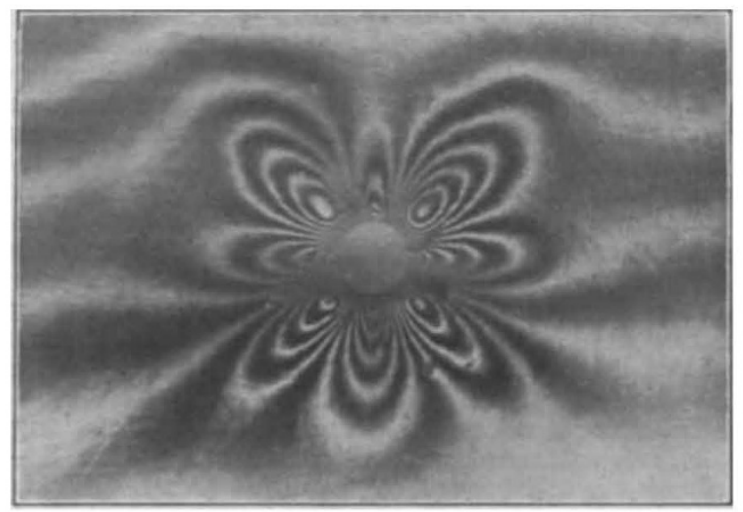

FIG. 1.

The loops, of course, are simply contours either of depressions or of elevations of the crystal surface. In Fig. 1, the borders are parallel to the sides of the elementary crystal cube; in this case, families of loops having axes parallel to the sides of the cube represent depressions, while those with axes parallel to the diagonals represent elevations.

Mount Wilson Observatory,

Sinclarr Smith.

Pasadena, California, April 23.

\section{Photographic Effects of Vitamins A and B.}

THE biological effect of vitamins is well known, but their chemical nature is most elusive. We have recently been investigating their physical effects, and some results obtained may be of interest.

Photographic plates were covered with aluminium foil and letters were cut out of the foil covering the glass side. Extracts of vitamins A and B, biologically tested, were used to paint the letters VA and VB on the glass side. The vitamin A used was ether extract of dried ox-liver, the solvent being removed in nitrogen. Vitamin B was water extract of purified brewers' yeast. The plates, wrapped in black paper, were left for three days ; on development, clear images of the letters were obtained.

To confirm the results, vitamins $\mathrm{A}$ and $\mathrm{B}$ were sealed in two separate glass tubes, and the experiment was repeated. Very sharp images were again obtained.

An extract of vitamin A prepared in a Paris research laboratory was investigated in the same way. It also gave positive results.

Vitamins destroyed but not carbonised did not affect the plates.

Two solutions, one ten times stronger than the other, of vitamin $A$ in paraffin oil and vitamin $B$ in water, were compared. The plates showed clearly difference in strength. Control experiments of pure solvents gave unfogged plates.

It is interesting to note that the effect of vitamin $B$ is similar to that of vitamin A, although the two vitamins are of different origin.
The experiments were repeated several times, and the same definite effect was present. We are proceeding with our research into these effects.

London.

Sophie Botcharsky.

Laboratoire d'Électrochimie,

Anna Foehringer.

École des Hautes Études, Paris.

\section{Isomeric Thiosulphuric Acids.}

IN continuation of an investigation of thiosulphatocobalt complexes, ${ }^{1}$ it has been found possible to isolate two very interesting isomeric modifications of thiosulphato-penta-cyano cobaltic acid $\mathrm{H}_{4}\left[(\mathrm{CN})_{5}\right.$. Co. $\left.\mathrm{S}_{2} \mathrm{O}_{3}\right]$; the difference between which lies in the constitution of the thiosulphate radical. The two acids differ in their chemical behaviour and form two corresponding series of crystalline isomeric salts. Both the complex acids hydrolyse in warm water, liberating two different varieties of free thiosulphuric acid. One of the latter decomposes as usual into sulphur dioxide and sulphur, whereas the other gives rise to sulphuretted hydrogen and sulphuric acid. This furnishes a positive and direct evidence of the existence of two isomeric forms of thiosulphuric acid, and has indeed led to their isola. tion in solution. The two different ways in which the constitution of thiosulphuric acid has been represented in many text-books correspond, therefore, in reality to these two isomeric forms. In fact, Piccard and Thomas ${ }^{2}$ obtained an indication of the synthesis of thiosulphuric acid from sulphur trioxide and hydrogen sulphide.

The constitution of the two forms is best represented by the well-known formulæ:<smiles>OO[Se](O)(O)O[Mg]</smiles>

Priyadaranjan Rây.

University College of Science, Calcutta, April 15.

I Rây, Jour. Ind. Chem. Soc., 4, 64, 326; 1927.

a Helv. Chim. Acta, 6, 1032 ; 1923 .

\section{Ravens Flying Upside-down.}

In NATURE of Dec. 20, 1930, p. 956, Mr. Sydney Evershed has described an observation relating to ravens flying upside-down. From this note one gets the impression that the habit is rather rare; from my observations, however, this is not the case. For some years I have had the opportunity of seeing many hundreds of these interesting birds in Iceland, where the number of individuals of this species apparently is rapidly increasing, probably because the refuse from the fisheries affords food for very large numbers of them. In some places - as Westmannaeyer and Hnifsdal in Isafjord-I have seen about a hundred of these birds performing evolutions in the air round favourite resting places, and here it is a rather common appearance to see the birds flying upside-down in the manner described, though I do not think I have seen them flying for so long a distance as 1000 metres, as recorded by Mr. Evershed. The upside-down flight is, so far as I can judge, not particularly connected with the courtship, though it is most often seen at the time of courtship, when air acrobatics are especially performed by the birds.

Copenhagen,

May 4.

No. 3214, VoL. 127] 